\title{
BANDWIDTH LIMITED AMPLIFICATION OF 220 fs PULSES IN XeCl: THEORETICAL AND EXPERIMENTAL STUDY OF TEMPORAL AND SPECTRAL BEHAVIOR
}

\author{
Bernhard DICK, Sandor SZATMÁRI, Bela RÁCZ and Fritz P. SCHÄFER \\ Max-Planck-Institut für biophysikalische Chemie, Abteiling Laserphysik, D.3400 Göttingen, Fed. Rep. Germany
}

Received 15 December 1986

\begin{abstract}
The spectral structure of the gain-profile of $\mathrm{XeCl}$ was found to strongly influence the spectral and temporal characteristics of amplified ultrashort light pulses. A simple model was used to numerically study both effects as a function of amplifier length and input pulse energy. The results are in good agreement with experiments. With these data the performance of a XeCl amplifier was optimized for shortest pulse duration with minimum distortion.
\end{abstract}

\section{Introduction}

In the preceding paper [1] Szatmári et al. reported on the generation of ultrashort light pulses at $308 \mathrm{~nm}$ and their subsequent amplification in a $\mathrm{XeCl}$ amplifier. The ultrashort pulses are generated at $616 \mathrm{~nm}$ in a distributed feedback dye laser (DFDL) and frequency doubled in a KDP crystal. These light pulses have a smooth spectrum with a width of ca. $50 \mathrm{~cm}^{-1}$ (see fig. 2 in [1]). The autocorrelation measurement of the DFDL light pulses at $616 \mathrm{~nm}$ yields a width of $500 \mathrm{fs}$ corresponding to a pulse duration of $320 \mathrm{fs}$ when a sech ${ }^{2}$ pulse shape is assumed. Hence we have strong evidence that the seed pulses at $308 \mathrm{~nm}$ are transform limited with a pulse duration of $200-250$ fs.

When amplification of these pulses in a $\mathrm{XeCl}$ amplifier was attempted, the spectrum of the amplified pulse displayed a modulation apparently reflecting the vibrational structure of the lasing transition in $\mathrm{XeCl}$. At the same time side peaks appeared in the autocorrelation traces located at $\pm 1.3 \mathrm{ps}$ from the central peak. Also, the central peak was considerably broadened. After a second pass through the $\mathrm{XeCl}$ amplifier the spectral as well as the temporal modulation strongly increased.

Through careful readjustment of the whole setup including exchange of all reflecting surfaces it was shown that the temporal modulation apparent from the autocorrelation is not due to a reflection but intrinsic to the amplifying process in $\mathrm{XeCl}$. Hence there was strong evidence that the temporal modulation is directly related to the spectral modulation. On the first sight this leads to a very pessimistic prognosis concerning the possibility to amplify light pulses of less than $250 \mathrm{fs}$ duration to high power levels with a $\mathrm{XeCl}$ amplifier.

In order to gain a better insight into the spectral and temporal evolution of ultrashort light pulses in an amplifier with structured gain profile we performed numerical calculations. The underlying simple model will be described in the following section. With this model we were able to reproduce the increasing temporal and spectral distortion of the light pulse with increasing length of the amplifier in good agreement with our observations.

Computer simulations with a fixed amplifier length and varying input pulse energy predicted a strong influence of the latter on the shape of the output light pulses, which was experimentally verified. With the combined experience from numerical simulations and experiments it was, finally, possible to find optimal operating conditions for the $\mathrm{XeCl}$ amplifier. Under these conditions amplified pulses of $5 \mathrm{~mJ}$ energy and 220 fs duration are obtained which show only slight modulation in their spectra and no sidebands in the autocorrelation traces. We believe that these pulses are close to the shortest pulses which can be achieved through amplification in $\mathrm{XeCl}$. 


\section{Numerical simulations}

We simulated the pulse spectra and autocorrelation traces which can directly be compared to experimental results. As starting point we took the well known equation of Frantz and Nodvik [2]

$$
W_{\mathrm{o}} / W_{\mathrm{s}}=\ln \left\{1+\left[\exp \left(W_{\mathrm{i}} / W_{\mathrm{s}}\right)-1\right] \exp \left(g_{0} L\right)\right\} \text {. }
$$

In this expression $W_{\mathrm{i}}$ is the input energy (per area) of the seed pulse fed into the amplifier, $W_{\mathrm{o}}$ is the corresponding energy of the output pulse, $W_{\mathrm{s}}$ is the saturation energy, and $g_{0}$ a constant gain coefficient. Eq. (1) has been derived with a rate-equation model neglecting the finite spectral width of the light pulse and the gain curve. Hence it is, strictly speaking, only applicable to homogeneously broadened amplifier media with phase-relaxation time $T_{2}$ much shorter then the duration of the light pulse to be amplified.

These conditions are not fulfilled for $\mathrm{XeCl}$ : Corkum and Taylor [3] have investigated the amplifier properties of $\mathrm{XeCl}$ with seed pulses of 2 ps duration and $10 \mathrm{~cm}^{-1}$ bandwidth. They could burn holes into the gain-profile which recovered with a time constant of $40 \mathrm{ps}$. Nevertheless they obtained a good fit of their data to eq. (1) with $W_{\mathrm{s}} \approx 1 \mathrm{~mJ} / \mathrm{cm}^{2}$. Recently Glownia et al. [4] performed a similar experiment with seed pulses of $350 \mathrm{fs}$ duration and

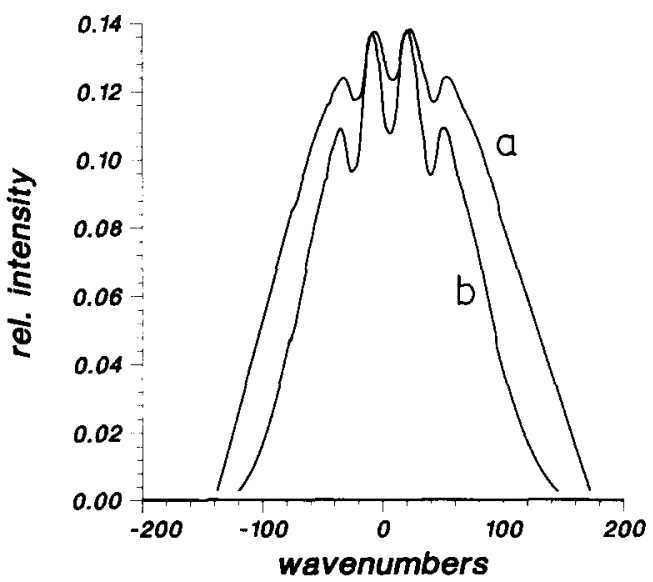

Fig. 1. Curve (a): uncorrected gain profile of $\mathrm{XeCl}$ adapted from the measurements of Corkum and Taylor [3]. Curve (b): corrected gain profile obtained from curve a through deconvolution with a gaussian with $10 \mathrm{~cm}^{-1}$ width. The wavenumber scale refers to a center frequency of $32468 \mathrm{~cm}^{-1}$, i.e. $308 \mathrm{~nm}$. obtained $W_{\mathrm{s}}=2.1 \mathrm{~mJ} / \mathrm{cm}^{2}$ and $g_{0}=0.13 \mathrm{~cm}^{-1}$. Corkum and Taylor used pulses of $1 \AA$ bandwidth and were thus able to measure the gain $g(\nu)$ as a function of frequency. This gain profile shows four maxima reflecting the vibrational structure of the $\mathrm{A} \rightarrow \mathrm{X}$ transition in $\mathrm{XeCl}$ (see fig. 1).

Our approach to the calculation of the spectral evolution of a light pulse during amplification is to use eq. (1) for each spectral component of the pulse and assume independent amplification of all spectral components. The latter assumption seems to be justified by the large inhomogeneous contribution to the bandwidth of the gain and the slow (40 ps) crossrelaxation.

Hence we replace the energies $W$ with the corresponding power spectra $I(\nu)$ and use a frequency dependent gain coefficient $g(\nu)$ :

$$
\begin{aligned}
& I_{\mathrm{o}}(\nu) / I_{\mathrm{s}}=\ln \left\{1+\left[\exp \left(I_{i}(\nu) / I_{\mathrm{s}}\right)-1\right]\right. \\
& \quad \times \exp (g(\nu) L)\} .
\end{aligned}
$$

For low input intensities this reduces to

$I_{\mathrm{o}}(\nu)=I_{\mathrm{i}}(\nu) \exp (g(\nu) L)$,

i.e. the output spectrum is the input spectrum modulated by the small signal gain. This fact was already observed by Glownia et al. [4].

To calculate the autocorrelation curves from these spectra we use the fact that the electric field $E(\nu)$ in frequency domain and $E(t)$ in time domain are related by the Fourier transform:

$E(\nu)=\int \mathrm{d} t E(t) \exp (\mathrm{i} 2 \pi \nu t)=: \mathscr{F}[E(t)]$,
$E(t)=\int \mathrm{d} \nu E(\nu) \exp (-2 \pi \mathrm{i} \nu t)=: \mathscr{F}^{-1}[E(\nu)]$.

The power spectrum is $I(\nu)=E(\nu) E^{*}(\nu)$, and the temporal intensity profile of the pulse is $I(t)=E(t) E^{*}(t)$. Note that $I(t)$ and $I(\nu)$ are not related by the Fourier transform as in eq. (3), since all phase information is lost in taking the absolute square. Hence there is no unique way to calculate the (slow) autocorrelation function

$A(t)=\int \mathrm{d} t^{\prime} I\left(t^{\prime}\right) I\left(t^{\prime}+t\right)$

from $I(\nu)$. Therefore, we must make an assumption concerning the phase in $E(\nu)$. We use the simplest approach $E(\nu)=\sqrt{I(\nu)}$. 
This approximation is equivalent to the assumption that the amplification process introduces no phase shift between various frequency components, and that a transform limited pulse remains transform-limited throughout the amplification process. For the numerical evaluation of the autocorrelation function we employed the convolution theorem of the Fourier transform leading to

$A(t)=\mathscr{F}^{-1}\left[J(\nu) J^{*}(-\nu)\right]$,

with

$J(\nu)=\mathscr{F}[(t)]$

With a Fast-Fourier-Transform routine [5] these calculations could be performed within a few seconds on a personal computer.

\section{Experimental}

The experimental setup used for the generation of ultrashort light pulses at $308 \mathrm{~nm}$ was the same as that described in ref. [1]. The energy of the light pulses injected into the second amplifier pass was varied with attenuators. In this way the response of the $\mathrm{XeCl}$-amplifier to short input pulses could be studied over a wide range of input-intensities approaching the saturation intensity. The first observations of the temporal and spectral pulse modulation mentioned in the introduction were obtained with a slightly different setup. There the arrangement to cut the ASE level consisted of two telescopes, resulting in much greater losses and hence reducing the input energy available for the second amplifier pass. The spectra of the output pulses were dispersed by a 3600 $\mathrm{l} / \mathrm{mm}$ grating and measured with a diode array. Autocorrelation traces of the ultrashort pulses were measured through two photon resonant multiphoton ionization in triethylamine as in ref. [1].

\section{Results and discussion}

For all simulations an input pulse at $308 \mathrm{~nm}$ with sech $^{2}$-shape and $200 \mathrm{fs}$ pulse width was assumed. The parameters varied were the length of the amplifier, $L$, and the ratio $I_{\mathrm{i}}(\max ) / I_{\mathrm{s}}=Q$.

From the wavelength-dependent gain measure- ments of Corkum and Taylor [3] we approximated the smooth gain-profile shown in fig. 1a. The maximum of this gain profile was scaled to a value of $g_{0}=0.138 \mathrm{~cm}^{-1}$ corresponding to a small signal gain of $\exp \left(g_{0} L\right) \approx 1000$ observed with an effective amplifier length of $L=50 \mathrm{~cm}$.

This gain profile has been obtained with a resolution of $1 \AA\left(10 \mathrm{~cm}^{-1}\right)$, which is not negligible compared to the separation of the maxima and minima in the gain profile. Hence this uncorrected gain curve considerably underestimates the difference in gain $\Delta g=g_{\max }-g_{\min }$ between the maxima and the minima in the gain curve. The simulations are, however, very sensitive to the value of $\Delta g$. Hence we used a corrected gain profile calculated through deconvolution of the uncorrected gain profile with a gaussian of 10 $\mathrm{cm}^{-1}$ width. This corrected gain profile is shown as curve (b) in fig. 1.

The simulated performance of the amplifier as a function of its length is shown in fig. 2. The input intensity was scaled to $Q=10^{-5}$. The spectrum and autocorrelation curve corresponding to this input pulse are displayed in the first row of fig. 2. The following rows show the corresponding characteristics of the pulse after passage through an amplifier of 33 $\mathrm{cm}, 100 \mathrm{~cm}$, and $200 \mathrm{~cm}$ length. At $33 \mathrm{~cm}$ amplifier length the spectrum shows considerable modulation due to the different gain at different frequencies. At the same time two sidebands appear in the autocorrelation curve ca. \pm 1 ps from the main peak. With increasing length of the amplifier the spectral modulation and the intensity of the sidebands in the autocorrelation curve increase strongly. This is evident from the third row in fig. 2, corresponding to $L=100 \mathrm{~cm}$. However, when the amplifier length is further increased, the spectrum of the pulse becomes broader with less modulation. At the same time the sidebands in the autocorrelation curve almost disappear.

This behavior can be easily understood on the basis of eq. (2). For input intensities much smaller than the saturation intensity eq. (2) can be rewritten as

$I_{\mathrm{o}}(\nu) / I_{\mathrm{s}}=\ln \left\{1+I_{\mathrm{i}}(\nu) / I_{s} \exp (g(\nu) L)\right\}$.

For amplifier lengths small enough to satisfy

$I_{\mathrm{i}}(\nu) / I_{\mathrm{s}} \exp (g(\nu) L) \ll 1$,

this reduces to eq. (3). In this limit the modulation 
Spectrum
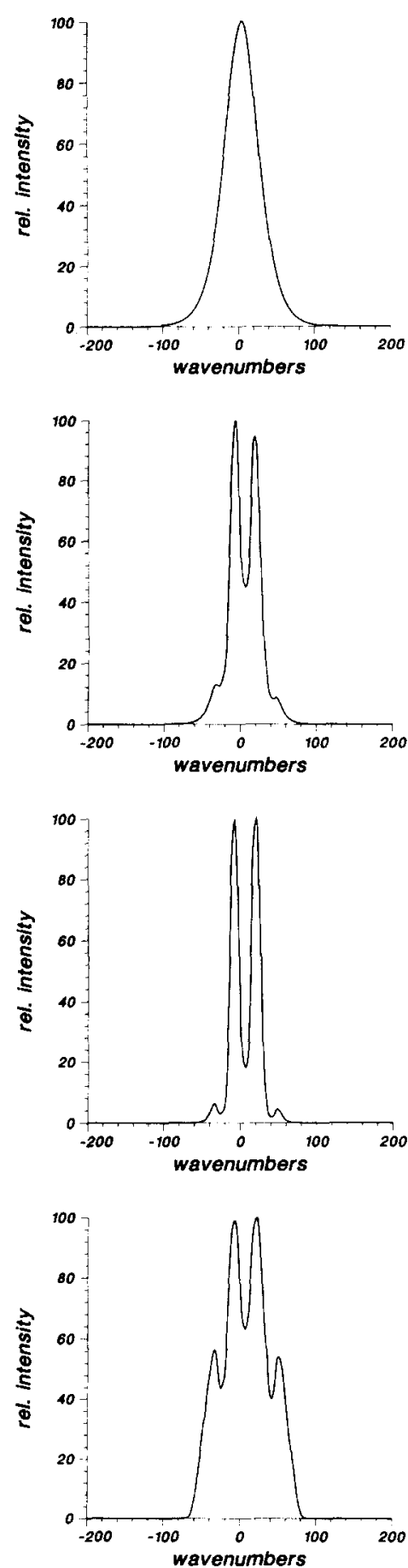

Autocorrelation
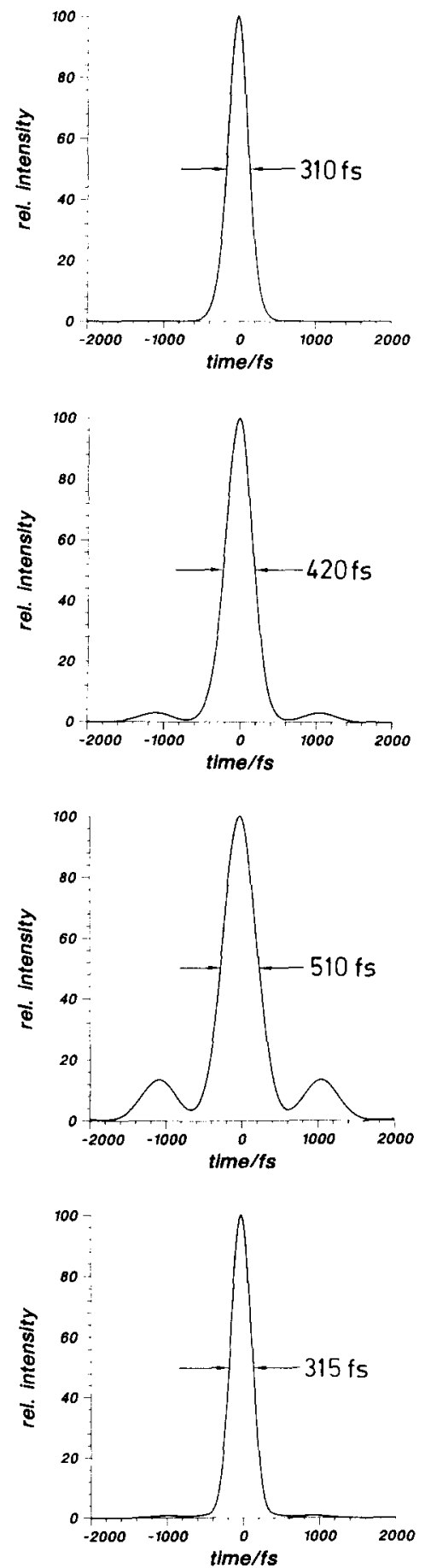

$L=0$

$L=33$

$L=100$

Fig. 2. Simulated amplifier performance as a function of length. Left column: power spectra of the light pulse, centered at $32468 \mathrm{~cm}$ $(308 \mathrm{~nm})$. Right column: corresponding autocorrelation curves. The first row refers to the input pulse which was approximated by a $\operatorname{sech}^{2}$-shape of $200 \mathrm{fs}$ duration and with intensity of $I_{\max } / I_{\mathrm{s}}=10^{-5}$. The following rows show the output pulse after passage through an amplifier of length $33 \mathrm{~cm}, 100 \mathrm{~cm}$, and $200 \mathrm{~cm}$, from top to bottom. 
Spectrum
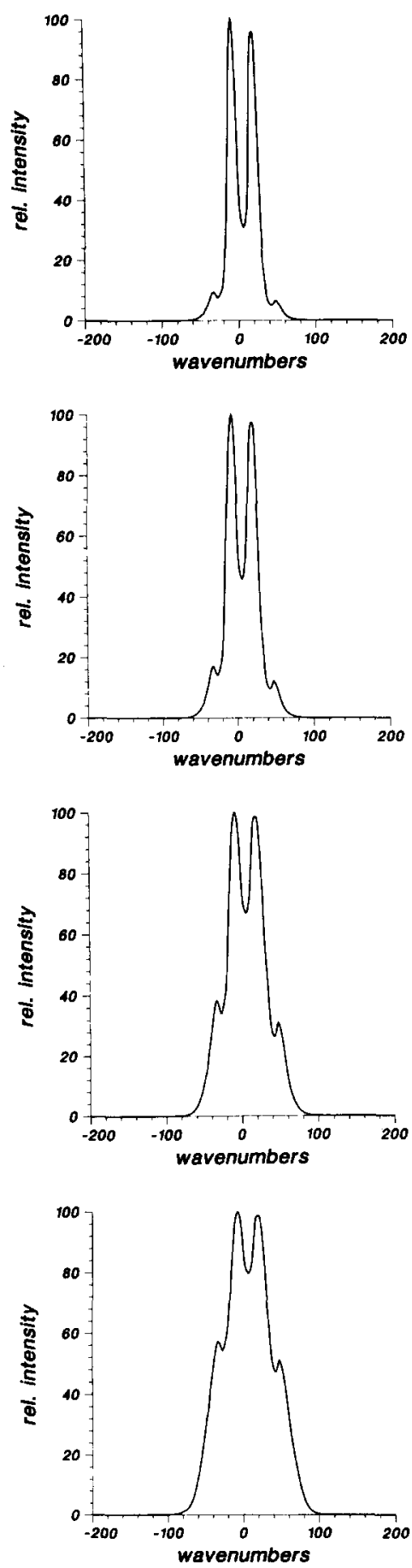

Autocorrelation
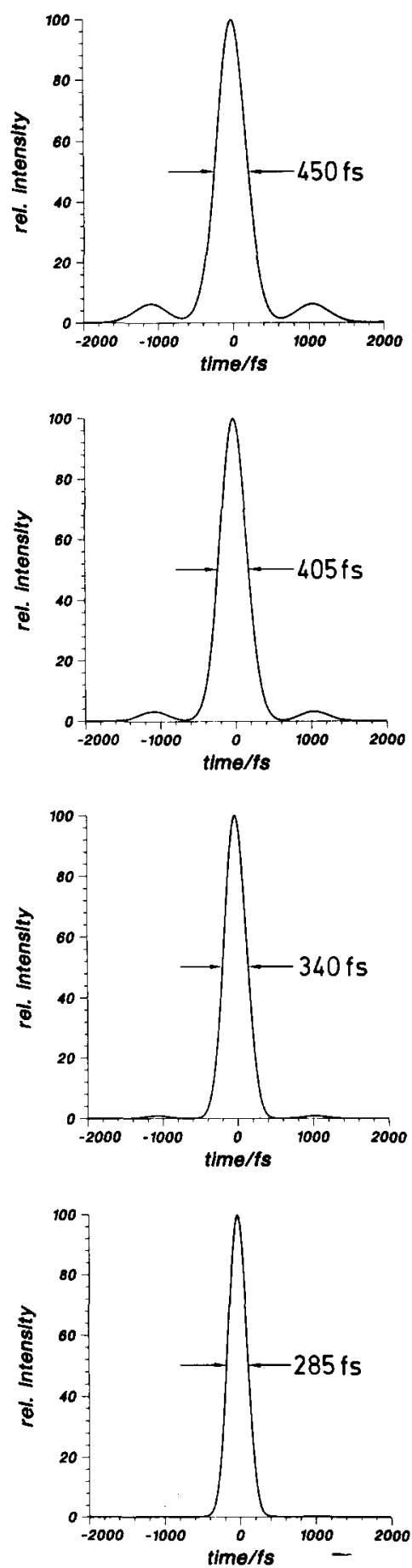

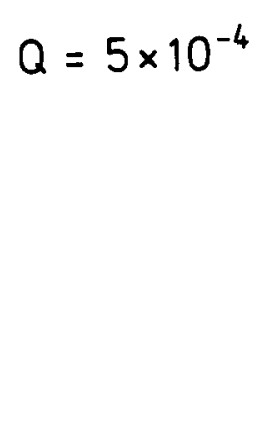

$Q=5 \times 10^{-3}$

$Q=5 \times 10^{-2}$

Fig. 3. Simulated amplifier performance as a function of input intensity for a fixed length of $50 \mathrm{~cm}$. Left column: power spectra of the amplified light pulses. Right column: corresponding autocorrelation curves. The input pulse had sech'shape with a pulse width of 200 fs. The input intensity $I_{\max } / I_{\mathrm{s}}$ from top to bottom is: $5 \times 10^{-4}, 5 \times 10^{-3}, 5 \times 10^{-2}, 5 \times 10^{-1}$. 

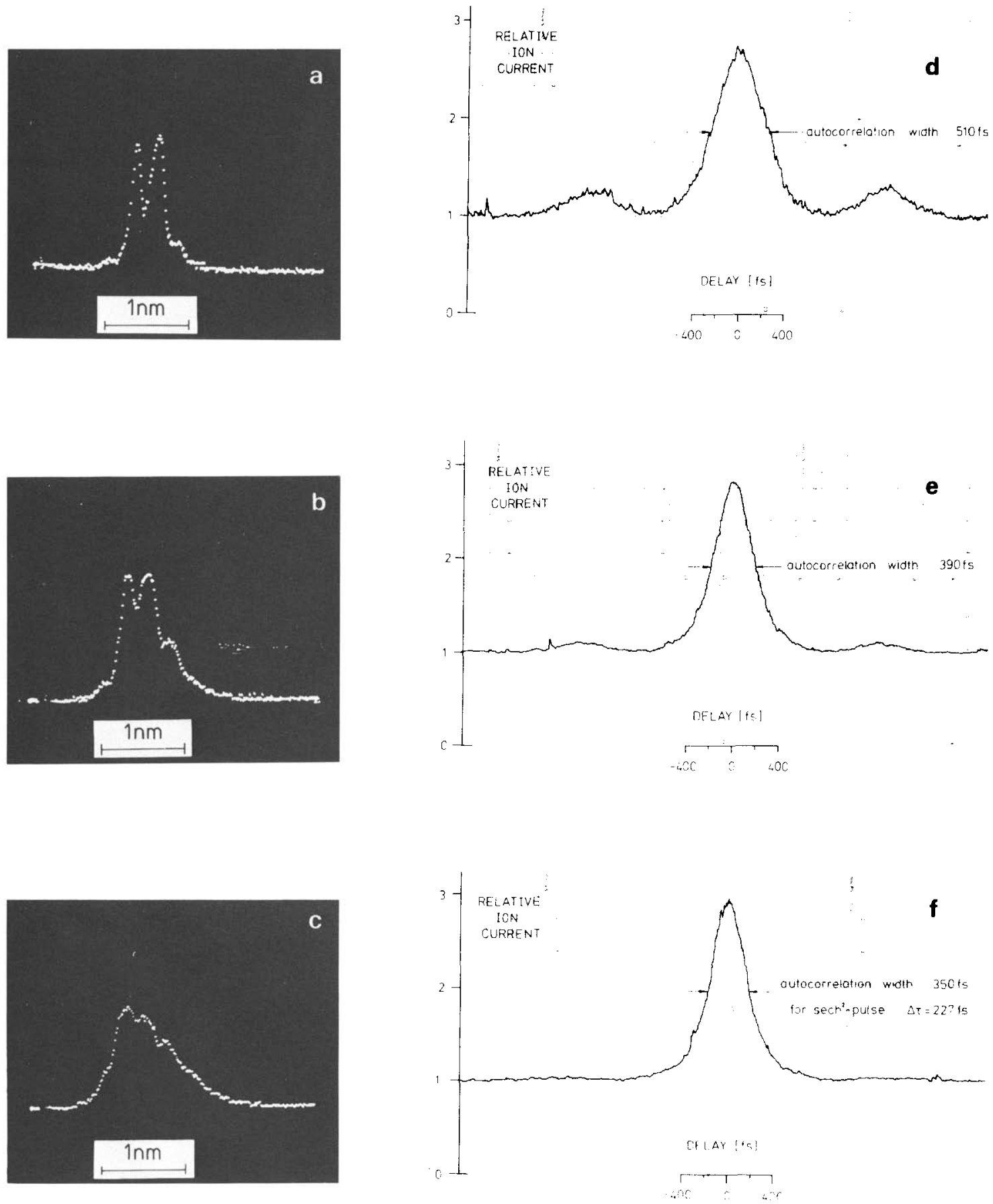

Fig. 4. Experimentally determined amplifier performance as a function of the input pulse energy. Left column: power spectra of the output pulse, right column: autocorrelation curves. The input energy from top to bottom is $1 \mu \mathrm{J}, 10 \mu \mathrm{J}$, and $100 \mu \mathrm{J}$. 
of the spectrum at the frequencies $\nu_{1}$ and $\nu_{2}$ of maximal and minimal gain respectively, increases exponentially with the length of the amplifier:

$\frac{I_{\mathrm{o}}\left(\nu_{1}\right)}{I_{\mathrm{o}}\left(\nu_{2}\right.}=\frac{I_{\mathrm{i}}\left(\nu_{1}\right)}{I_{\mathrm{i}}\left(\nu_{2}\right)} \exp (\Delta g L)$

This behavior changes when saturation sets in. Then eq. (8) reduces to

$I_{\mathrm{o}}(\nu) / I_{\mathrm{s}}=\ln \left(I_{\mathrm{i}}(\nu) / I_{s}\right)+g(\nu) L$.

Hence for long amplifiers the pulse spectrum will approach the gain profile $g(\nu)$. The reason for this is the fact that the spectral components in the maxima of the gain curve will be saturated at shorter amplifier lengths than those at the minima, leading to stronger amplification of the valley of the spectrum with further amplification. We can estimate that this effect sets in a saturation length $L_{\mathrm{s}}$ for which

$g_{\max } L_{\mathrm{s}}=-\ln \left(I_{\mathrm{i}}\left(\nu_{1}\right) / I_{\mathrm{s}}\right)$.

At this point the largest modulation of the spectrum will be obtained.

This largest modulation is proportional to $\exp \left(\Delta g L_{\mathrm{s}}\right)$. Hence a shorter $L_{\mathrm{s}}$ will lead to much smaller modulation, and in order to achieve this, the input intensity must be increased. The input spectrum has its maximum at $\nu_{2}$ (the minimum of the gain curve) and $70 \%$ of this value at $\nu_{1}$ (the maximum of the gain curve). Hence for the pulse used in fig. 2 with $Q=10^{-5}$ we obtain $L_{\mathrm{s}}=86 \mathrm{~cm}$ and $\exp \left(\Delta g L_{\mathrm{s}}\right)=12.1$. Increasing the input intensity to $Q=5 \times 10^{-4}$ reduces $L_{\mathrm{s}}$ to $58 \mathrm{~cm}$ and the maximum modulation to 5.3. Hence for a given length $L$ of the amplifier the input energy must be high enough to yield $L_{s}<L$ in order to reduce the spectral and temporal distortion of the pulse.

The behavior of an amplifier with a fixed length of $50 \mathrm{~cm}$ to input pulses of increasing energy is simulated in fig. 3 . The same pulse shape as in fig. 2 was used as input. The first row of fig. 3 corresponds to $Q=5 \times 10^{-4}$ and $L_{\mathrm{s}}=58 \mathrm{~cm}$. Under this condition the maximum distortion in the spectrum and the autocorrelation are observed. The width of the central peak in the autocorrelation curve also indicates a lengthening of the pulse duration by $50 \%$. The following rows show the results for $Q=5 \times 10^{-3}$, $5 \times 10^{-2}$, and $5 \times 10^{-1}$ corresponding to $L_{\mathrm{s}}=41 \mathrm{~cm}$, $24 \mathrm{~cm}$, and $7.6 \mathrm{~cm}$, respectively. With increasing input intensity the spectrum becomes broader and less modulated. The sidebands in the autocorelation curves decrease in intensity, and the central peak becomes narrower.

The situation simulated in fig. 3 was experimentally realized through attenuation of the input pulse injected into the second pass of the $\mathrm{XeCl}$ amplifier. We approximated the normalized input intensity $Q$ with the ratio of the input energy to the saturation energy density. The latter was taken to be $2.1 \mathrm{~mJ} / \mathrm{cm}^{2}$ as measured by Glownia et al. [4] with subpicosecond pulses. (Preliminary experiments in our laboratory indicate that the saturation energy might be somewhat lower.) Fig. 4 shows the results obtained with injected pulse energies of $1 \mu \mathrm{J}, 10 \mu \mathrm{J}$, and 100 $\mu \mathrm{J}$ (unattenuated), corresponding to $Q=5 \times 10^{-4}$, $5 \times 10^{-3}$, and $5 \times 10^{-2}$, respectively. These experimental results agree very well with the simulations: With increasing input energy the distortion of the spectrum is reduced, the sidebands in the autocorrelation curve disappear, and the pulse duration is compressed.

This observation is strong evidence that the approximations in our simple model are justified. Since broadening of the spectrum and reduction of its modulation is accompanied by pulse-shortening it is also likely that the light pulse remains transform-limited during amplification.

\section{Acknowledgement}

This work was supported in part by the Bundesministerium für Forschung und Technologie and by the Deutsche Forschungsgemeinschaft through its Leibniz-program.

\section{References}

[1] S. Szatmári, B. Rácz and F.P. Schäfer, Optics Comm. 62 (1987) 271.

[2] L.M. Frantz and J.S. Nodvik, J. Appl. Phys. 34 (1963) 2346.

[3] P.B. Corkum and R.S. Taylor, IEEE J. Quantum Electron. QE-18 (1982) 1962.

[4] J.H. Glownia, G. Arjavalingam, P.P. Sorokin and J.E. Rothenberg, Optics Lett. 11 (1986) 79.

[5] E.O. Brigham, The fast Fourier transform (Prentice-Hall, Englewood Cliffs, New Jersey, 1974). 